\title{
Assessing Myometrial Infiltration by Endometrial Cancer: Uterine Virtual Navigation with Three- dimensional US ${ }^{1}$
}

Juan Luis Alcázar, MD

Rosendo Galván, MD

Sonia Albela, MD

Sergio Martinez, MD

Jaume Pahisa, MD

Matías Jurado, MD

Guillermo López-García, MD
Purpose:

Materials and Methods:

Results: Ninety-six women (mean age, 61.8 years; range, 31-86

Conclusion:

1 From the Department of Obstetrics and Gynecology, Clínica Universitaria de Navarra, University of Navarra, Avenida Pio XII, 36, 31008 Pamplona, Spain (J.L.A., R.G., M.J., G.L.); and Department of Obstetrics and Gynecology, Hospital Clinic, University of Barcelona, Barcelona, Spain (S.A., S.M., J.P.). Received May 19, 2008; revision requested July 3; revision received July 10; accepted August 4; final version accepted September 16. Address correspondence to J.L.A. (e-mail: jlalcazar@unav.es).

๑) RSNA, 2009 years) with endometrial cancer were included in the study. At histologic analysis, myometrial invasion was found to be less than $50 \%$ in $69(72 \%)$ cases and $50 \%$ or more in 27 (28\%) cases. TDS measured with US was positively correlated with histologically measured TDS $(r=0.649 ; 95 \%$ confidence interval: $0.52,0.76)$. The best cutoff for USmeasured TDS was $9.0 \mathrm{~mm}$ (sensitivity, 100\%; specificity, $61 \%$; negative predictive value, $100 \%$; positive predictive value, $50 \%$ ). Subjective impression had a sensitivity of $92.6 \%$, a specificity of $82.3 \%$, a negative predictive value of $96.6 \%$, and a positive predictive value of $67.7 \%$.

To describe and analyze the diagnostic performance of uterine virtual navigation with three-dimensional (3D) ultrasonography (US) for the assessment of the depth of myometrial infiltration by endometrial cancer.

Institutional review board approval was obtained; patients gave oral informed consent. Women with endometrial cancer were evaluated by using 3D US prior to surgical staging. A 3D volume of the whole uterus was obtained and analyzed by using software. Virtual navigation through by analyzing the lateral, anterior, posterior, and fundal portions of the myometrium. Myometrial infiltration was also assessed by subjective impression of an examiner. Histologic findings of myometrial infiltration and TDS meated to identify the best cutoff for TDS for identifying myometrial infiltration of $50 \%$ or more.

Uterine virtual navigation with 3D US is a reliable method for the assessment of myometrial infiltration in patients with endometrial cancer.

๑ RSNA, 2009 
E ndometrial carcinoma is the most frequent gynecologic malignancy (1). Since 1988, the International Federation of Gynecology and Obstetrics has recommended surgical staging as the initial treatment in these patients to determine the extent of disease. This implies total abdominal hysterectomy with bilateral salpingo-oophorectomy, peritoneal cytologic samplings, and pelvic and paraaortic lymphadenectomy (2). However, complete routine staging has come under criticism in cases of low risk of lymph node involvement (3-5). Low-risk patients are mainly defined with three histologic factors-tumor type, tumor histologic grade, and myometrial infiltration (3). For this reason, many authors advocate intraoperative gross or frozen section evaluation of myometrial invasion and tumor grade in clinical stage I endometrial carcinoma to aid in the decision of whether lymphadenectomy should be performed or could be obviated $(6,7)$. Frozen section analysis has been shown to be highly accurate (94\%) for determining myometrial invasion (8). However, frozen section analysis may be time consuming, and it is not performed in all hospitals. On the other hand, gross evaluation has been shown to be much less accurate (9-13) than frozen section analysis. Therefore, a method that could reliably assess myometrial invasion preoperatively would be helpful to provide individual tailoring of the surgical approach.

Several imaging techniques performed prior to surgery are currently used for the assessment of the depth of myometrial infiltration by endometrial cancer. Contrast material-enhanced magnetic resonance (MR) imaging is considered the most reliable method and has an accuracy ranging from $62 \%$ to $95 \%$ (14-20). However, MR imaging is costly, has limited availability, and cannot be performed in all patients.

\section{Advance in Knowledge}

- With uterine virtual navigation and three-dimensional US, we were able to predict deep myometrial infiltration in all cases.
Some groups $(13,21-26)$ have assessed the role of transvaginal twodimensional (2D) ultrasonography (US) for the assessment of myometrial invasion with considerable variation in methods and results. This could be explained by the fact that this technique is highly operator dependent because it is mainly based on the subjective impression of the examiner.

Recently, three-dimensional (3D) US has been introduced in clinical practice. This allows unique ways for assessing the uterus, such as virtual navigation through multiplanar display and tomographic US imaging (27). This technique has been demonstrated to be much less operator dependent than 2D US $(28,29)$.

The purpose of our study was to describe and analyze the diagnostic performance of uterine virtual navigation with 3D US for the assessment of the depth of myometrial infiltration by endometrial cancer.

\section{Materials and Methods}

\section{Patients}

Institutional review board (Clínica Universitaria de Navarra) approval was obtained before starting the study, and all women gave verbal informed consent. From January 2003 to December 2007, 113 consecutive women with clinical stage I endometrial cancer (cancer confined to the uterine corpus according to results of physical examination, chest radiography, and abdominal and pelvic CT scanning) were recruited for this prospective observational study (Fig 1). Patients were from two centers-Clínica Universitaria de Navarra, Pamplona, Spain ( $n=78,69 \%$ ), and Clinic Hospital, Barcelona, Spain $(n=35,31 \%)$.

\section{Implication for Patient Care}

- Three-dimensional US with uterine virtual navigation offers a relatively simple, reliable, and reproducible way for preoperative assessment of myometrial infiltration in patients with endometrial cancer.
All patients had histologically proved endometrial cancer according to results of office microcurettage or hysteroscopic biopsy and were scheduled for surgical staging.

Exclusion criteria were as follows: surgery not performed $(n=4)$; surgery performed in hospital other than that of recruitment $(n=5)$; incomplete pathologic report $(n=2)$; and incomplete $3 \mathrm{D}$ volume with exclusion of uterine serosal margins, including one case that also did not have a reference standard $(n=6)$. Three premenopausal women with childbearing desire with well-differentiated focal endometrioid carcinoma associated with endometrial hyperplasia with atypia and no signs of myometrial infiltration at MR imaging and who were considered to have stage IA G1 endometrioid carcinoma were offered fertility-sparing treatment. These patients did not undergo surgical staging. However, we did not exclude them in our analysis and considered them to have no myometrial infiltration for analytic purposes on the basis of MR imaging results, in spite of the absence of histologic data.

\section{US Examination}

All US examinations were performed within 1 week prior to surgery, with patients in the lithotomy position and with an empty bladder by using a scanner (Voluson 730 Expert; GE Healthcare, Milwaukee, Wis) equipped with a multifrequency endovaginal probe (3-9 $\mathrm{MHz}$ ). Three operators (J.L.A., R.G.,

\section{Published online before print 10.1148/radiol.2503080877 \\ Radiology 2009; 250:776-783 \\ Abbreviations: \\ TDS = tumor-free distance to serosa \\ $3 \mathrm{D}=$ three-dimensional \\ $2 \mathrm{D}=$ two-dimensional}

\section{Author contributions:}

Guarantors of integrity of entire study, J.L.A., R.G., S.A.; study concepts/study design or data acquisition or data analysis/interpretation, all authors; manuscript drafting or manuscript revision for important intellectual content, all authors; manuscript final version approval, all authors; literature research, J.L.A.; clinical studies, all authors; statistical analysis, J.L.A.; and manuscript editing, J.L.A.

Authors stated no financial relationship to disclose. 
and S.A., with 7, 3, and 1 year of experience in $3 \mathrm{D}$ US, respectively) performed all examinations according to a predetermined scanning protocol (29). A 2D US exploration of the uterus and adnexa was initially performed. Endometrial thickness was measured in the sagittal plane, including both layers, at the level of maximal thickness. Thereafter, the 3D volume box was activated and was manually adjusted to include the entire uterus. With a sweep angle of $90^{\circ}$ and with the patient remaining as still as possible, a $3 \mathrm{D}$ volume was acquired. Acquisition time lasted from 6 to 10 seconds. Once the volume was acquired, it was stored and evaluated later with a personal computer.

\section{Stored Volume Analysis}

Two examiners (J.L.A. and S.A.) performed all volume examinations in consensus and were blinded to histologic results. Myometrial infiltration was assessed off-line in the stored volume by using software (4DView, version 5.0; GE Healthcare). By using the "selected planes" utility, we performed rotations until uterine transverse, sagittal, and coronal planes were placed on planes A, $\mathrm{B}$, and $\mathrm{C}$, respectively, on the computer screen (Fig 2). Virtual navigation through the uterus then was performed. By first going through plane B (sagittal) from the anterior to the posterior uterine serosa, we navigated through plane $\mathrm{C}$ (coronal). By using the image with the subjective thickest endometrial distance, we measured the myometrial TDS in the lateral wall and fundus (Fig 3).

We then navigated in plane $\mathrm{C}$ (coronal) from one uterine cornu to the other. By using the image with the subjective thickest endometrial distance in plane B (sagittal), we measured TDS in the anterior and posterior uterine walls (Fig 4).

To determine myometrial infiltration depth, we selected the subjective shortest TDS found in any area. Only one measurement per area was obtained.

Myometrial infiltration depth was also estimated subjectively by the examiners according to their impression of depth of invasion $(>50 \%$ or $<50 \%)$ during virtual navigation. This was performed by looking at the point in which myometrial-endometrial interface was not clearly identified and then by looking at the supposedly tumor-free myometrial wall at this point. By using the opposite myometrial wall as a comparison, if a marked asymmetry was found, deep (>50\%) infiltration was stated; if myometrial thickness was similar in both myometrial walls, superficial $(<50 \%)$ infiltration was stated.
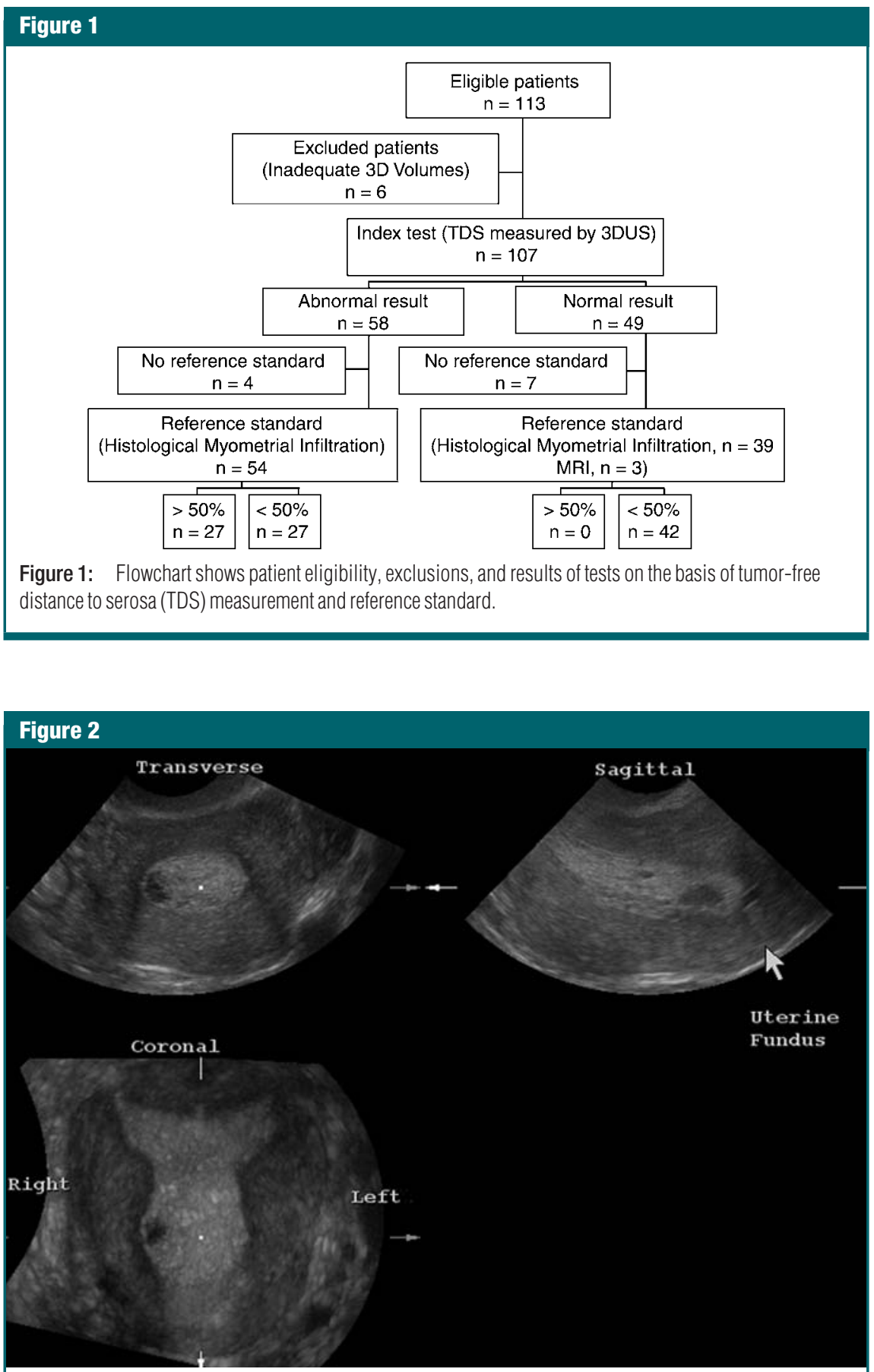

Figure 2: Three-dimensional US images in the uterus in a case of endometrial cancer. 


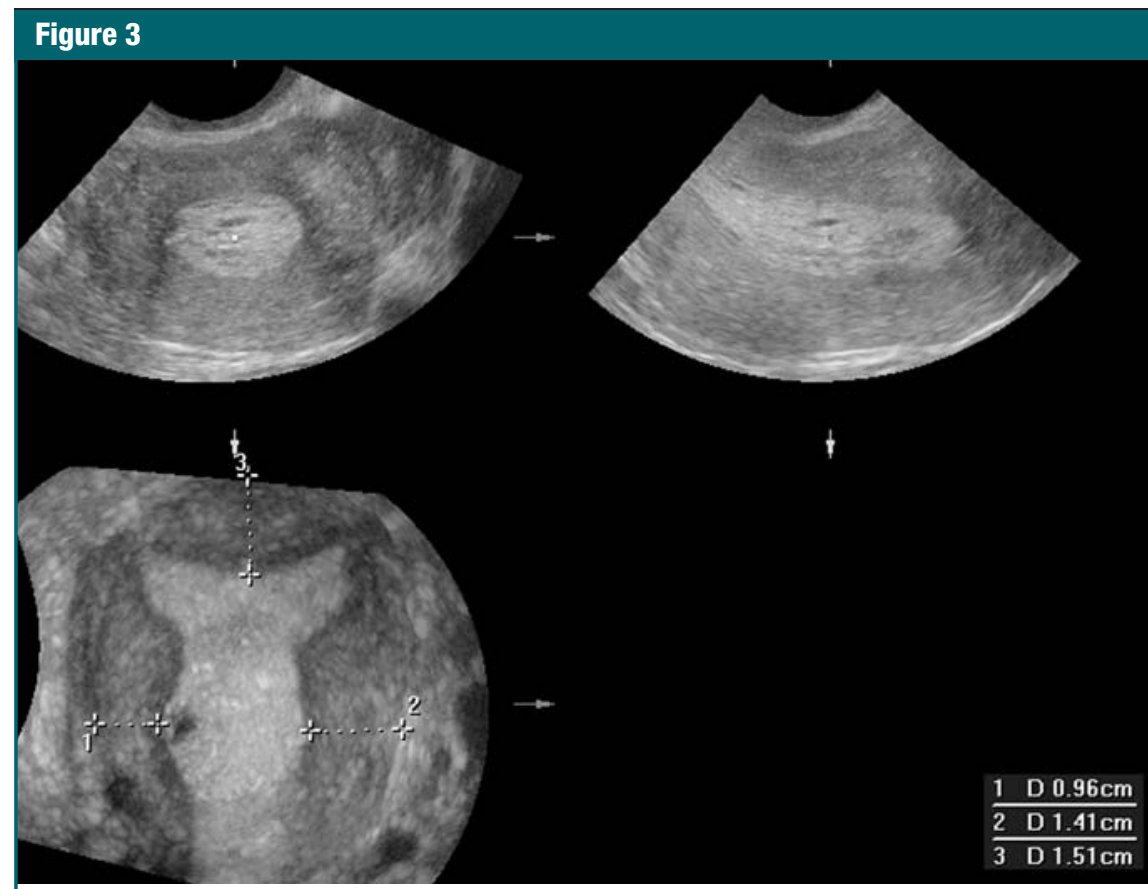

Figure 3: Three-dimensional US images (transverse, top left; sagittal, top right; coronal, bottom left) show measurement of TDS in coronal plane $(9.6 \mathrm{~mm}$ in right uterine wall, $14.1 \mathrm{~mm}$ in left uterine wall, and 15.1 in fundus). $D=$ distance.

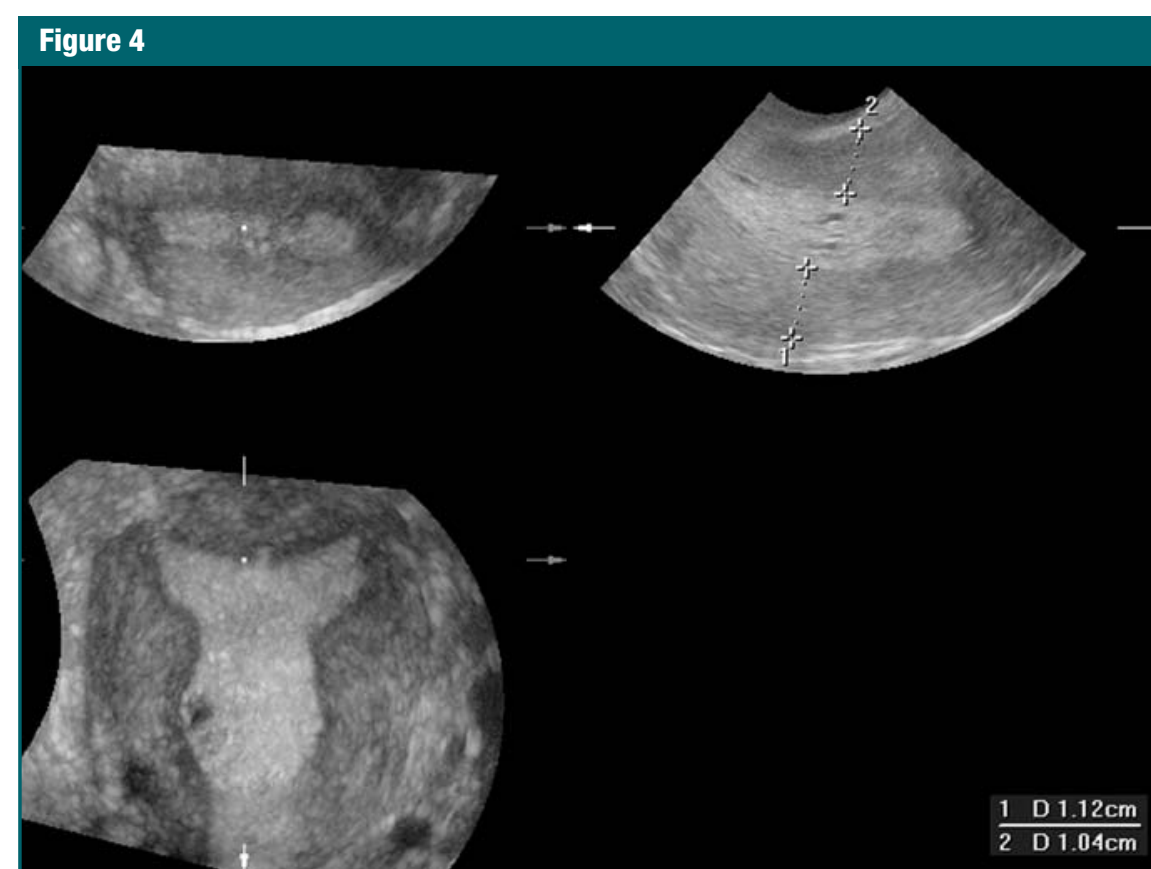

Figure 4: Three-dimensional US images (transverse, top left; sagittal, top right; coronal, bottom left) show measurement of TDS in sagittal plane (11.2 $\mathrm{mm}$ in anterior uterine wall and $10.4 \mathrm{~mm}$ in posterior uterine wall). $D=$ distance.
Cervical involvement was stated to be present when there was subjective disruption of the cervical canal.

Uterine myomas were noted, and the subjective effect of myomas on myometrial measurements was assessed.

\section{Control Subjects}

To determine normal TDS in uteri without pathologic examination results, we used two control groups of asymptomatic women. One group included premenopausal women $(n=20)$, and the other group included postmenopausal women $(n=20)$. We defined menopause as 1 year of absence of menstruation in women older than 45 years.

None of these women had subjective uterine or endometrial pathologic conditions (leiomyomas, adenomyosis, congenital anomalies, endometrial polyps, hyperplasia, or endometrial thickness $>$ $4 \mathrm{~mm}$ in postmenopausal women). None of these women were taking hormonal treatments, such as contraceptives, hormone replacement therapy, or tamoxifen citrate.

\section{Reproducibility Studies}

Reproducibility of TDS measurements was assessed in the first 15 volumes. One examiner (J.L.A.) analyzed the same volume twice, with the second analysis being performed at least 1 week after the first analysis. To determine interobserver reproducibility, a second examiner (R.G.) analyzed the same 15 volumes. Examiners were blinded to each other's reviews when performing analysis. These evaluations were performed with one examiner of the pair different than those who evaluated the volume by consensus.

\section{Surgical Procedure and Tumor Staging}

Surgery included total abdominal hysterectomy with bilateral salpingo-oophorectomy, peritoneal washings, omentectomy, and pelvic and paraaortic lymphadenectomy. After surgery, the pathologic report included histologic type, histologic grade and myometrial infiltration depth, lymphvascular space involvement, TDS, and lymph node metastases. These data were used as the reference standard. All surgeries were performed by gynecological on- 


\section{Table 1}

\begin{tabular}{|c|c|}
\hline \multicolumn{2}{|c|}{$\begin{array}{l}\text { Histologic Features of Endometrial } \\
\text { Cancer }\end{array}$} \\
\hline Parameter & No. of Patients \\
\hline \multicolumn{2}{|l|}{ Histologic type } \\
\hline Endometrioid & $80(83)$ \\
\hline Adenosquamous & $8(8)$ \\
\hline Serous papillary & $6(6)$ \\
\hline Clear cell & $2(2)$ \\
\hline \multicolumn{2}{|l|}{ Histologic grade } \\
\hline G1 & $42(44)$ \\
\hline G2 & $31(32)$ \\
\hline G3 & $23(24)$ \\
\hline \multicolumn{2}{|c|}{ Myometrial infiltration } \\
\hline None ${ }^{\star}$ & $10(10)$ \\
\hline$<50 \%$ & $59(61)$ \\
\hline$\geq 50 \%$ & $27(28)$ \\
\hline \multicolumn{2}{|l|}{ Tumor stage } \\
\hline IA & $10(10)$ \\
\hline IB & $48(50)$ \\
\hline IC & $14(15)$ \\
\hline$\|$ & $8(8)$ \\
\hline III & $16(17)$ \\
\hline
\end{tabular}

Note.-Data in parentheses are percentages. Percentages may not add up to $100 \%$ because of rounding.

* In three cases, no myometrial infiltration was considered on the basis of MR imaging results.

cologists (M.J., G.L., J.L.A., J.P., and S.M., each with more than 10 years of experience in gynecological oncology surgery).

Tumor stage was determined according to International Federation of Gynecology and Obstetrics criteria (2). Tumor histologic grade was determined according to the following three-grade system: Grade 1 carcinoma showed solid growth pattern in less than $5 \%$ of the tumor. Grade 2 carcinoma showed solid growth pattern in 5\%$50 \%$ of the tumor. Grade 3 carcinoma showed solid growth pattern in more than $50 \%$ of the tumor.

\section{Statistical Analysis}

The Kolmogorov-Smirnov test was used to assess normal distribution of continuous data. Continuous data were compared by using one-way analysis of variance when data were normally distributed or by using the Mann-Whitney $U$ test when data were not normally distributed. These tests were used for comparing TDS measured by using US in the control group according to the site of measurement, TDS measured by using US in the control subject and study groups,

\section{Table 2}

\section{Measurement of TDS in Control Groups according to Site of Measurement}

\begin{tabular}{llll} 
Control Group & Anterior and Posterior Uterine Walls $(\mathrm{mm})^{*}$ & ${\text { Lateral Uterine Wall }(\mathrm{mm})^{\dagger}}^{\dagger}$ & Fundus $(\mathrm{mm})^{\ddagger}$ \\
\hline Premenopausal & $17(11-22)$ & $15(11-20.5)$ & $13(8-16)$ \\
Postmenopausal & $15(9-21)$ & $13(11-22)$ & $11(10-18)$ \\
\hline
\end{tabular}

Note.-Data are medians, with ranges in parentheses.

${ }^{*} P=.224$, Mann-Whitney $U$ test.

${ }^{\dagger} P=.195$, Mann-Whitney $U$ test.

$\ddagger P=.026$, Mann-Whitney $U$ test.

and TDS measured by using US in study group according to histologic depth of invasion.

The Wilcoxon signed rank test was used for comparing TDS measured by using US and TDS measured by a pathologist. Categoric variables were compared by using a $\chi^{2}$ test. The Spearman correlation coefficient $(r)$ was used to assess the correlation between TDS measured by using US and TDS measured by a pathologist. A receiver operating characteristic curve was plotted to determine the best cutoff value for TDS for identifying deep myometrial invasion. The best cutoff value was chosen according to the best sensitivity with the lowest false-positive rate. Positive and negative likelihood ratios were also calculated.

Sensitivity and specificity of TDS measured by using US and those measured by using subjective impression of infiltration depth were compared by using the $\mathrm{McNe}-$ mar test.

This was a convenience sample. Because no previous data were available for determining a significant difference in sensitivity or specificity for this technique, we were not able to estimate a sample size properly.

Reproducibility was assessed by using the intraclass and interclass correlation coefficients according to the BlandAltman method (30).

A $P$ value of .05 or less was considered to indicate a statistically significant difference. All analyses were performed by using software (SPSS, version 15.0; SPSS, Chicago, IIl).

\section{Results}

Patient mean age was 61.8 years, ranging from 31 to 86 years. Eighty-five $(89 \%)$ women were postmenopausal,

\section{Table 3}

TDS Measured by Using US and by a Pathologist according to Myometrial Infiltration

\begin{tabular}{|c|c|c|}
\hline Parameter & $\begin{array}{l}\text { US-Measured } \\
\text { TDS }(\mathrm{mm})^{\star}\end{array}$ & $\begin{array}{l}\text { Histologically } \\
\text { Measured TDS } \\
(\mathrm{mm})^{\dagger}\end{array}$ \\
\hline $\begin{array}{l}\text { Myometrial } \\
\text { infiltration }\end{array}$ & & \\
\hline$<50 \%$ & $10(2-20)$ & $15(6-35)$ \\
\hline$\geq 50 \%$ & $4(1-8)$ & $4(0-16)$ \\
\hline $\begin{array}{l}\text { Control } \\
\text { subjects }\end{array}$ & $12(8-22)$ & NA \\
\hline
\end{tabular}

Note.-Data are medians, with ranges in parentheses. $\mathrm{NA}=$ not applicable.

${ }^{*} P<.001$ for comparison among all three groups (myometrial infiltration $<50 \%$, myometrial infiltration $\geq 50 \%$, and control subjects), according to the Mann-Whitney $U$ test.

$\dagger p<.001$ for myometrial infiltration of less than $50 \%$ versus that of $50 \%$ or more, according to the MannWhitney $U$ test.

and 11 (11\%) women were premenopausal.

Tumor histologic features are shown in Table 1.

Median endometrial thickness for the study group was $13.0 \mathrm{~mm}$ (interquartile range, 10.0; range, 1.0-49.0 $\mathrm{mm})$. Median TDS at the level of fundus, anterior and posterior uterine walls, and lateral uterine wall in the control groups are shown in Table 2 . TDS in all uterine walls was longer in premenopausal women than in postmenopausal women.

Median TDS measured by a pathologist and that measured by using US were significantly shorter in those tumors with $50 \%$ or more myometrial infiltration than in those with less than 
$50 \%$ infiltration (Table 3 ). There was a positive correlation between pathologically measured and US-measured TDS $(r=0.649 ; 95 \%$ confidence interval: $0.52,0.76)$. Median TDS measured by using US (7.0 mm; range, 1-20 mm) was significantly shorter than that measured by a pathologist $(11.0 \mathrm{~mm}$; range, $0-35 \mathrm{~mm})(P<.001)$ (Fig 5).

Median TDS in control groups was significantly longer than in the study group for tumors infiltrating less than $50 \%$ and for those infiltrating more than $50 \%$ (Table 3 ).

The receiver operating characteris-

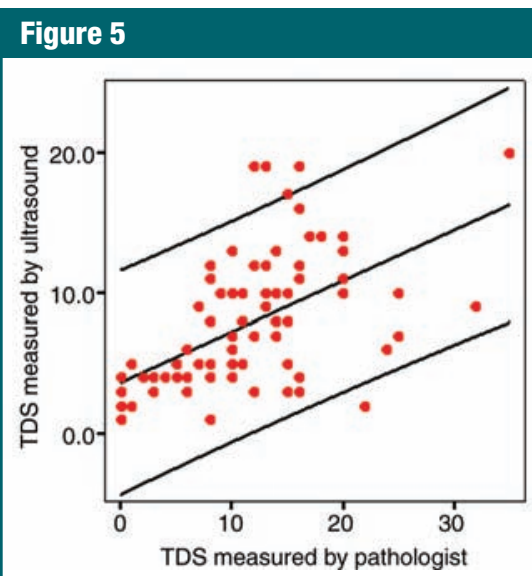

Figure 5: Scatterplot shows TDS measured by using US versus that measured by a pathologist.

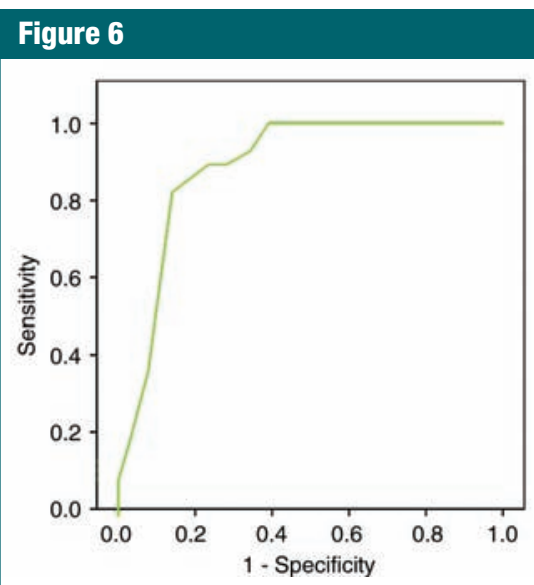

Figure 6: Receiver operating characteristic curve for TDS. Area under the curve for TDS was 0.89 (95\% confidence interval: $0.82,0.95)$. Best cutoff for TDS was $9.0 \mathrm{~mm}$. tic curve showed that shorter US-measured TDS was a predictor for deep myometrial infiltration (Fig 6). The best cutoff value for US-measured TDS was $9.0 \mathrm{~mm}$, which allowed for identification of all cases of deep infiltration with no false-negative cases. However, by applying this cutoff, a $39 \%$ false-positive rate was obtained. Figure 7 shows a case of deep myometrial infiltration as determined by using 3D US.

One case in the control group had a TDS of $8.0 \mathrm{~mm}$.

Sensitivity, specificity, positive predictive value, negative predictive value, and likelihood ratio for TDS and subjective impression are shown in Tables 4 and 5 . With subjective impression, the

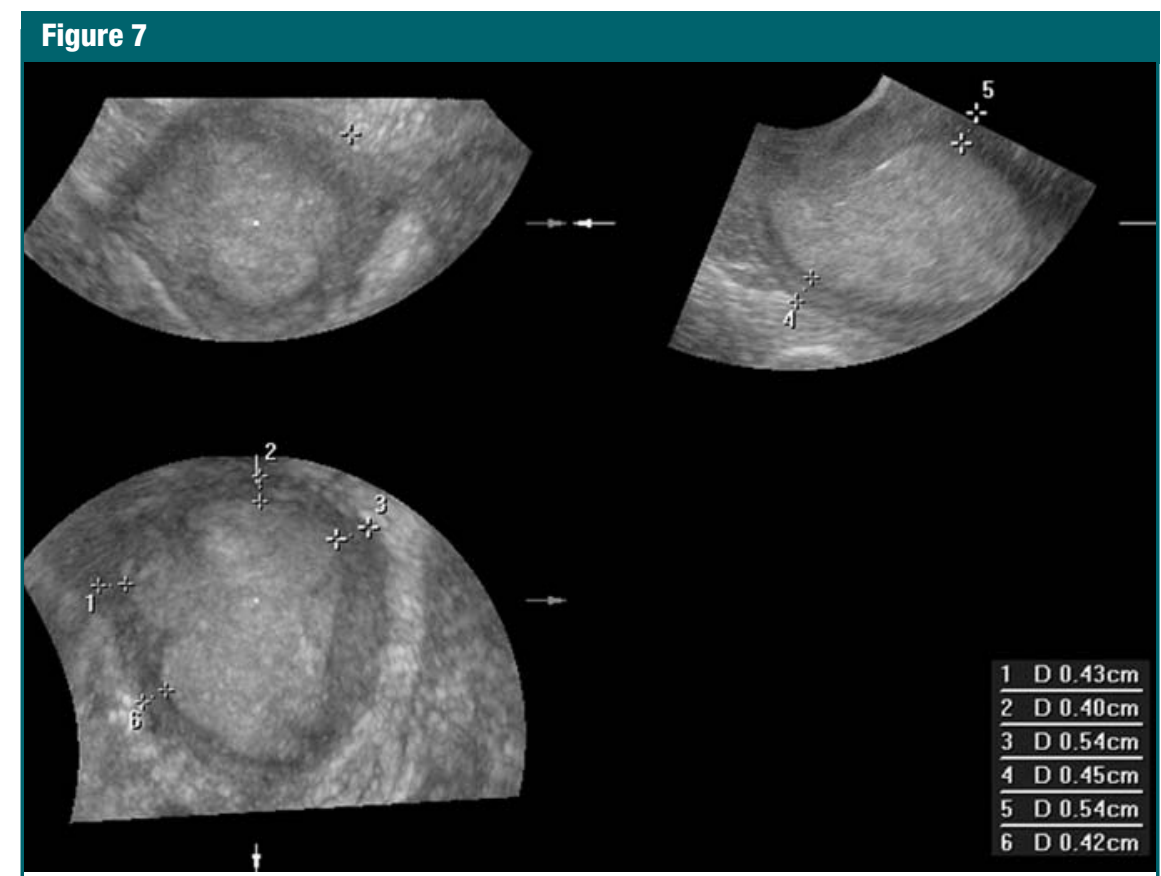

Figure 7: Three-dimensional US images show deep myometrial infiltration. The shortest TDS was $4.0 \mathrm{~mm}$ in the fundus. $D=$ distance.

\section{Table 4}

\section{Diagnostic Performance of Shortest TDS Measured by Using US}

\begin{tabular}{llrl} 
& \multicolumn{2}{c}{$\begin{array}{c}\text { Myometrial } \\
\text { Infiltration }\end{array}$} & \\
\cline { 2 - 3 } Parameter & $<50 \%$ & $\geq 50 \%$ & Total \\
\hline TDS & & & \\
$>9.0 \mathrm{~mm}$ & 42 & 0 & 42 \\
$\leq 9.0 \mathrm{~mm}$ & 27 & 27 & 54 \\
Total & 69 & 27 & 96 \\
\hline
\end{tabular}

Note.-Sensitivity was 100\% (95\% confidence interval: $87.5 \%, 100 \%)$. Specificity was $61 \%(95 \%$ confidence interval: $48 \%, 71.5 \%)$. Positive predictive value was $50 \%$. Negative predictive value was $100 \%$. Positive likelihood ratio was 2.56 (95\% confidence interval: $1.90,3.43)$.

\section{Table 5}

Diagnostic Performance of Subjective Impression of Examiner

\begin{tabular}{cccc} 
& \multicolumn{2}{c}{$\begin{array}{c}\text { Myometrial } \\
\text { Infiltration }\end{array}$} & \\
\cline { 2 - 3 } Parameter & $<50 \%$ & $\geq 50 \%$ & Total \\
\hline Subjective & & & \\
impression & & & \\
$<50 \%$ & 57 & 2 & 59 \\
$\geq 50 \%$ & 12 & 25 & 37 \\
\hline Total & 69 & 27 & 96 \\
\hline
\end{tabular}

Sensitivity was $92.6 \% \quad(95 \%$ confidence interval: $76.6 \%, 97.9 \%)$. Specificity was $82.6 \%$ (95\% confidence interval: $72.0 \%, 89.8 \%$ ). Positive predictive value was $67.6 \%$. Negative predictive value was $96.6 \%$. Positive likelihood ratio was 5.32 (95\% confidence interval: $3.15,9.00)$. Negative likelihood ratio was $0.09(95 \%$ confidence interval: $0.02,0.34$ ). 
false-positive rate was $17.4 \%$, which was much lower than that of TDS $(P<$ $.001)$. However, sensitivity was also lower at $92.6 \%$.

Myomas were found in 26 (27\%) patients. In most of the cases, the location was subserous. In five cases, the presence affected the measurement of TDS. In three of these cases, myometrial infiltration was overestimated because the examiner measured from the myometrial-endometrial interface to the inner border of the myoma, resulting in a TDS shorter than what it actually was.

Intraobserver and interobserver reproducibility for US TDS measurement were high, with intraclass correlation coefficients of 0.967 and 0.912 , respectively.

Cervical involvement was correctly identified in seven of eight $(88 \%)$ cases of stage II endometrial carcinoma.

\section{Discussion}

Myometrial infiltration is one of the most important factors associated with lymph node metastases (31) in endometrial carcinoma. In spite of International Federation of Gynecology and Obstetrics recommendations about surgical staging in endometrial cancer, the role of comprehensive lymphadenectomy in cases of endometrioid histologic type $(32,33)$ remains controversial. The key question is whether systematic pelvic and paraaortic lymphadenectomy is beneficial in all patients or could be safely obviated in selected cases. Therefore, reliable estimation of myometrial infiltration is essential for deciding whether to perform lymph node dissection. Most gynecologic oncologists rely on intraoperative gross or frozen section pathologic analysis once the uterus has been removed. However, gross evaluation has a sensitivity of $67 \%-77 \%$, which means a false-negative rate for detection of deep myometrial invasion of about $23 \%-33 \%$ (10-14).

MR imaging and transvaginal 2D US have been proposed for preoperative evaluation of myometrial infiltration by endometrial cancer. In many institu- tions, MR imaging has become a standard method. Sensitivity reported for this technique ranges from $80 \%$ to $91 \%$ (16-21).

Three-dimensional US allows virtual navigation by using multiplanar display and tomographic US imaging, which renders images in planes similar to those seen at MR imaging (28). This technique has been demonstrated to be much less operator dependent than 2D US (29).

A study by Lindauer et al (34) demonstrated that TDS has a significant prognostic importance for predicting recurrence in endometrial cancer. On the basis of this study, we speculated that we could reliably measure this distance by using 3D US and performing a virtual navigation throughout the uterus to identify the shortest myometrial TDS. This should be performed in all orthogonal planes because tumors may infiltrate any region of the myometrium.

Assessment of the sagittal plane by using $2 \mathrm{D}$ US is the traditional image plane utilized, but the coronal plane is frequently impossible to visualize by using 2D US. However, 3D US allows the assessment of the uterine coronal plane in almost all circumstances.

We found a significant positive correlation between TDS measured by using US and that measured by a pathologist. The presence of myomas may affect the reliability of TDS measurement because they may be a source of falsepositive cases. This could be considered a shortcoming of this technique. However, similar problems have been found when using MR imaging (35).

Our data show that a $9.0-\mathrm{mm}$ cutoff for TDS can accurately identify all cases of deep infiltration with no false-negative cases. By applying this cutoff, a $39 \%$ false-positive rate was obtained. This relatively high false-positive rate is a shortcoming for this technique in clinical practice.

Another limitation for this measurement was that healthy women may have TDS less than $9.0 \mathrm{~mm}$.

With subjective impression, the false-positive rate was $17.4 \%$, which is much lower; however, sensitivity decreased to $92.6 \%$. Therefore, it could be argued that subjective impression has a better performance than 3D US. However, because it is important to perform surgery only once, false-positive cases are less relevant than falsenegative cases from the point of view of the oncologist. Therefore, we believe that this technique could be helpful in those cases in which MR imaging is not available or cannot be performed.

In summary, we have shown that virtual navigation with $3 \mathrm{D}$ US is reproducible and helps identify all cases of deep myometrial infiltration. However, future larger prospective studies are needed to define its actual role in clinical practice.

\section{References}

1. Creasman WT, Odicino F, Maisonneuve P, et al. Carcinoma of the corpus uteri. Int $\mathrm{J}$ Gynaecol Obstet 2006;95(suppl 1):S105S143.

2. Shepherd JH. Revised FIGO staging for gynaecological cancer. Br J Obstet Gynaecol 1989;96:889-892.

3. Aalders JG, Thomas G. Endometrial cancer: revisiting the importance of pelvic and para aortic lymph nodes. Gynecol Oncol 2007; 104:222-231

4. Chan JK, Cheung MK, Huh WK, et al. Therapeutic role of lymph node resection in endometrioid corpus cancer: a study of 12,333 patients. Cancer 2006;107:1823-1830.

5. Chan JK, Lin YG, Monk BJ, Tewari K, Bloss JD, Berman ML. Vaginal hysterectomy as primary treatment of endometrial cancer in medically compromised women. Obstet Gynecol 2001;97:707-711.

6. Homesley HD. Management of endometrial cancer. Am J Obstet Gynecol 1996;174:529534.

7. Rose PG. Endometrial cancer. N Engl J Med 1996;335:640-649.

8. Noumoff JS, Menzin A, Mikuta J, Lusk EJ, Morgan M, LiVolsi VA. The ability to evaluate prognostic variables on frozen section in hysterectomies performed for endometrial carcinoma. Gynecol Oncol 1991;42:202208.

9. Franchi M, Ghezzi F, Melpignano M, et al. Clinical value of intraoperative gross examination in endometrial cancer. Gynecol Oncol 2000;76:357-361.

10. Vorgias G, Hintipas E, Katsoulis M, Kalinoglou N, Dertimas B, Akrivos T. Intraoperative gross examination of myometrial inva- 
sion and cervical infiltration in patients with endometrial cancer: decision-making accuracy. Gynecol Oncol 2002;85:483-486.

11. Cunha TM, Félix A, Cabral I. Preoperative assessment of deep myometrial and cervical invasion in endometrial carcinoma: comparison of magnetic resonance imaging and gross visual inspection. Int J Gynecol Cancer 2001;11:130-136.

12. Traen K, Hølund B, Mogensen O. Accuracy of preoperative tumor grade and intraoperative gross examination of myometrial invasion in patients with endometrial cancer. Acta Obstet Gynecol Scand 2007;86:739741.

13. Berretta R, Merisio C, Piantelli G, et al. Preoperative transvaginal ultrasonography and intraoperative gross examination for assessing myometrial invasion by endometrial cancer. J Ultrasound Med 2008;27:349-355.

14. Manfredi R, Mirk P, Maresca G, et al. Localregional staging of endometrial carcinoma: role of MR imaging in surgical planning. Radiology 2004;231:372-378.

15. Sanjuán A, Cobo T, Pahisa J, et al. Preoperative and intraoperative assessment of myometrial invasion and histologic grade in endometrial cancer: role of magnetic resonance imaging and frozen section. Int J Gynecol Cancer 2006;16:385-390.

16. Chung HH, Kang SB, Cho JY, et al. Accuracy of MR imaging for the prediction of myometrial invasion of endometrial carcinoma. Gynecol Oncol 2007;104:654-659.

17. Vasconcelos C, Félix A, Cunha TM. Preoperative assessment of deep myometrial and cervical invasion in endometrial carcinoma: comparison of magnetic resonance imaging and histopathologic evaluation. J Obstet Gynaecol 2007;27:65-70.

18. Rockall AG, Meroni R, Sohaib SA, et al. Evaluation of endometrial carcinoma on magnetic resonance imaging. Int J Gynecol Cancer 2007;17:188-196.
19. Torricelli P, Ferraresi S, Fiocchi F, et al. 3-T MRI in the preoperative evaluation of depth of myometrial infiltration in endometrial cancer. AJR Am J Roentgenol 2008;190:489495.

20. Park JY, Kim EN, Kim DY, et al. Comparison of the validity of magnetic resonance imaging and positron emission tomography/ computed tomography in the preoperative evaluation of patients with uterine corpus cancer. Gynecol Oncol 2008;108:486-492.

21. Georgiev DB, Chernev T, Netzov V, Dimova DN. Preoperative sonographic evaluation of patients with endometrial carcinoma. Int J Gynaecol Obstet 1994;47:147-150.

22. Teefey SA, Stahl JA, Middleton WD, et al. Local staging of endometrial carcinoma: comparison of transvaginal and intraoperative sonography with gross visual inspection. AJR Am J Roentgenol 1996;166:547-552.

23. Alcázar JL, Jurado M, López-García G. Comparative study of transvaginal ultrasonography and CA 125 in the preoperative evaluation of myometrial invasion in endometrial carcinoma. Ultrasound Obstet Gynecol 1999;14:210-214

24. Arko D, Takac I. High frequency transvaginal ultrasonography in preoperative assessment of myometrial invasion in endometrial cancer. J Ultrasound Med 2000;19:639643.

25. Köse G, Aka N, Api M. Preoperative assessment of myometrial invasion and cervical involvement of endometrial cancer by transvaginal ultrasonography. Gynecol Obstet Invest 2003;56:70-76.

26. De Smet F, De Brabanter J, Van den Bosch $\mathrm{T}$, et al. New models to predict depth of infiltration in endometrial carcinoma based on transvaginal sonography. Ultrasound $\mathrm{Ob}-$ stet Gynecol 2006;27:664-671.

27. Benacerraf BR, Shipp TD, Bromley B. Improving the efficiency of gynecologic sonog- raphy with 3-dimensional volumes: a pilot study. J Ultrasound Med 2006;25:165-171.

28. Raine-Fenning NJ, Campbell BK, Clewes JS, Kendall NR, Johnson IR. The reliability of virtual organ computer-aided analysis (VOCAL) for the semiquantification of ovarian, endometrial and subendometrial perfusion. Ultrasound Obstet Gynecol 2003;22:633-639.

29. Alcázar JL, Mercé LT, Manero MG, Bau S, López-García G. Endometrial volume and vascularity measurements by transvaginal 3-dimensional ultrasonography and power Doppler angiography in stimulated and tumoral endometria: an interobserver reproducibility study. J Ultrasound Med 2005;24: 1091-1098.

30. Bland JM, Altman DG. Statistical methods for assessing agreement between two methods of clinical measurement. Lancet 1986;1: 307-310.

31. Creasman WT, Morrow CP, Bundy BN, Homesley HD, Graham JE, Heller PB. Surgical pathologic spread patterns of endometrial cancer: a Gynecologic Oncology Group study. Cancer 1987;60:2035-2040.

32. Chan JK, Kapp DS. Role of complete lymphadenectomy in endometrioid uterine cancer. Lancet Oncol 2007;8:831-841.

33. Chan JK, Wu H, Cheung MK, Shin JY, Osann K, Kapp DS. The outcomes of 27,063 women with unstaged endometrioid uterine cancer. Gynecol Oncol 2007;106:282-288.

34. Lindauer J, Fowler JM, Manolitsas TP, et al. Is there a prognostic difference between depth of myometrial invasion and the tumorfree distance from the uterine serosa in endometrial cancer? Gynecol Oncol 2003;91: 547-551.

35. Rieck GC, Bulman J, Whitaker R, Leeson SC. A retrospective review of magnetic resonance imaging in assessing the extent of myometrial infiltration for patients with endometrial carcinoma. J Obstet Gynaecol $2005 ; 25: 765-768$. 